Research Article

\title{
Neutrosophic Number Optimization Models and Their Application in the Practical Production Process
}

\author{
Angyan Tu $\mathbb{D}^{1,2}$ Jun $\mathrm{Ye}^{2}$ and Bing Wang $\mathbb{D}^{1}$ \\ ${ }^{1}$ School of Mechatronic Engineering and Automation, Shanghai University, 99 Shangda Road, Shanghai 200444, China \\ ${ }^{2}$ Department of Electrical and Information Engineering, Shaoxing University, 508 Huancheng West Road, Shaoxing, \\ Zhejiang 312000, China \\ Correspondence should be addressed to Bing Wang; susanbwang@shu.edu.cn
}

Received 15 December 2020; Revised 30 March 2021; Accepted 3 April 2021; Published 19 April 2021

Academic Editor: Broumi Said

Copyright (C) 2021 Angyan Tu et al. This is an open access article distributed under the Creative Commons Attribution License, which permits unrestricted use, distribution, and reproduction in any medium, provided the original work is properly cited.

In order to simplify the complex calculation and solve the difficult solution problems of neutrosophic number optimization models (NNOMs) in the practical production process, this paper presents two methods to solve NNOMs, where Matlab built-in function "fmincon()" and neutrosophic number operations (NNOs) are used in indeterminate environments. Next, the two methods are applied to linear and nonlinear programming problems with neutrosophic number information to obtain the optimal solution of the maximum/minimum objective function under the constrained conditions of practical productions by neutrosophic number optimization programming (NNOP) examples. Finally, under indeterminate environments, the fit optimal solutions of the examples can also be achieved by using some specified indeterminate scales to fulfill some specified actual requirements. The NNOP methods can obtain the feasible and flexible optimal solutions and indicate the advantage of simple calculations in practical applications.

\section{Introduction}

Traditional inventory models [1-4] and production planning models [5-7] involve deterministic constrained functions and/or objective functions in deterministic environments. Nevertheless, uncertainty is nearly universal in real world. Therefore, many uncertain optimization methods were proposed for optimization problems with uncertain variables, interval numbers, stochastic, and fuzzy logics [8-15]. In many applied fields, such as management, engineering, and design problems, uncertain programming has been broadly carried out so far. In order to obtain the optimal crisp values of the objective function and the optimal feasible crisp solutions of the decision variables, the constrained functions and/or objective functions are usually changed into some crisp or deterministic programming problems in existing uncertain programming approaches. So, the aforementioned transformed methods are not really meaningful indeterminate approaches because the real indeterminate optimization problems can only indicate indeterminate solutions rather than optimal crisp solutions in indeterminate environments. Nevertheless, indeterminate programming problems imply the corresponding indeterminate optimal values of the objective function and indeterminate optimal solutions for the decision variables under indeterminate environments. So, it is necessary to find some fit optimization approaches for dealing with indeterminate programming problems with indeterminate solutions.

Smarandache [16-18] is a pioneer of indeterminacy theories which provide the new minds to solve indeterminacy problems. He adopted the imaginary value denoted by $I$ and then introduced a neutrosophic number (NN) $z=x+y I$ for $x, y \in R$ ( $R$ : the set of all real numbers) composed of the determinate part $x$ and indeterminate part $y I$. As for describing indeterminate and incomplete information, obviously, NNs in the indeterminacy theories are a useful mathematical tool. With the development of indeterminacy theories, NNs were also applied to fault diagnosis [19, 20] and decision making $[21,22]$ under indeterminate environments. 
Further, thick function or interval function named neutrosophic function, neutrosophic precalculus, and neutrosophic calculus were provided by Smarandache [23] in 2015 , where thick function $e: S \longrightarrow E(S)(E(S)$ is the set of all interval functions) as the form of an interval function $e(x)=$ $\left[e_{1}(x), e_{2}(x)\right]$. The indeterminate function was applied in engineering problems successfully. For example, Ye et al. $[24,25]$ and Chen et al. [26, 27] proposed expressions of neutrosophic function and applied NNs in analyzing the joint roughness coefficient. Later, Ye [28] used neutrosophic linear equations of NNs to solve traffic flow problems.

At present, neutrosophic linguistic numbers, hesitant neutrosophic linguistic numbers, and their aggregation operators were applied to multiattribute decision making [29-31].

But in real situations, affected by each kind subjective and objective reasons, such as absences of precise information judged by decision makers or experts, loss of data, and measurement errors, there exist some indeterminate problems. As for the concepts of NNs, NN functions containing indeterminacy $I$ can represent the indeterminate problems with partial certainty and partial uncertainty under indeterminate environments. Ye [32] and Jiang and Ye [33] introduced NN nonlinear and linear programming models and their preliminary solution methods. However, existing methods for solving complex NN optimization problems imply some difficulty and calculational complexity in their solution process. Inspired by the previous solution methods, this paper first selects the models of practical applications in production process, such as inventory models and production planning models. Then, NN nonlinear and linear mathematical models and their solution methods (Matlab built-in function "fmincon()" and operations of NNs) are built with indeterminacy $I$ as our preliminary application study. Finally, real examples of NN linear programming (NN-LP) and NN nonlinear programming (NN-NP) problems illustrate the feasibility of the proposed methods. The advantage of the proposed methods is that the optimization calculations are simple and effective in practical applications.

The remainder of this paper is organized as follows. Section 2 depicts some concepts and their operations of NNs. Section 3 first introduces NN-NP problems with an inventory mathematical model and model formation and then uses two methods (Matlab built-in function "fmin$\operatorname{con}($ )" and operations of NNs) to solve the NN-NP problems in indeterminate setting. Section 4 presents NN-LP problems with the production planning mathematical model and model formation and then applies two methods regarding the Matlab built-in function "fmincon()" and operations of NNs to solve the solutions in the NN-NP problems and to show the simplicity and effectiveness of the proposed NN-LP methods. Conclusions and future research are provided in Section 5 .

\section{Mathematical Preliminaries}

2.1. Some Concepts and Their Operations of Neutrosophic Numbers (NNs). The concept of NN was first proposed by
Smarandache [34, 35], which consists of two parts (a determinate part and an indeterminate part). He defined the mathematical expression form $z=x+y I$ for $x, y \in R$, where $R$ represents all real numbers and $I$ is indeterminacy. So, it is conveniently used in indeterminate environments.

For example, consider that a $\mathrm{NN}$ is $z=13+5 I$. Then, its determinate part value is 13 and its indeterminate part value is $5 I$. When $I \in[0,0.5]$, it is equivalent to $z \in[13,15.5]$ for sure $z \geq 13$.

Let $z_{1}=x_{1}+y_{1} I$ and $z_{2}=x_{2}+y_{2} I$ be two NNs. Then, Smarandache $[34,35]$ gave their operations of NNs in the following:

(1) $z_{1}+z_{2}=x_{1}+x_{2}+\left(y_{1}+y_{2}\right) I$.

(2) $z_{1}-z_{2}=x_{1}-x_{2}+\left(y_{1}-y_{2}\right) I$.

(3) $z_{1} \times z_{2}=x_{1} x_{2}+\left(x_{1} y_{2}+x_{2} y_{1}+y_{1} y_{2}\right) I$, in particular, when $z_{1}=0$ and $z_{2}=I$, we get the equation with $0 \times I=0$.

(4) $z_{1}^{2}=\left(x_{1}+y_{1} I\right)^{2}=x_{1}^{2}+\left(2 x_{1} y_{1}+y_{1}^{2}\right) \mathrm{I}$, in particular, when $z_{1}=I$, we get the equation with $I 2=I$.

(5) $z_{1} / z_{2}=x_{1}+y_{1} I / x_{2}+y_{2} I=x_{1} / x_{2}+x_{2} y_{1}-$ $x_{1} y_{2} / x_{2}\left(x_{2}+y_{2}\right) I$ for $x_{2} \neq 0$ and $x_{2} \neq-y_{2}$.

(6) $\sqrt{z_{1}}=\sqrt{x_{1}+y_{1} I}=\left\{\begin{array}{l}\sqrt{x_{1}}-\left(\sqrt{x_{1}}+\sqrt{x_{1}+y_{1}}\right) I \\ \sqrt{x_{1}}-\left(\sqrt{x_{1}}-\sqrt{x_{1}+y_{1}}\right) I \\ -\sqrt{x_{1}}+\left(\sqrt{x_{1}}+\sqrt{x_{1}+y_{1}}\right) I \\ -\sqrt{x_{1}}+\left(\sqrt{x_{1}}-\sqrt{x_{1}+y_{1}}\right) I\end{array}\right.$.

\subsection{Example}

There are two NNs $z_{1}=5+3 I$ and $z_{2}=2+5 I$. Then, we can obtain the following results according to the above operations:

(1) $z_{1}+z_{2}=x_{1}+x_{2}+\left(y_{1}+y_{2}\right) I=5+2+(3+5) I=7+8 I$.

(2) $z_{1}-z_{2}=x_{1}-x_{2}+\left(y_{1}-y_{2}\right) I=5-2+(3-5) I=3-2 I$.

(3) $z \quad{ }_{1} \times z_{2}=x_{1} x_{2}+\left(x_{1} y_{2}+x_{2} y_{1}+y_{1} y_{2}\right) I=5 \times 2+(5 \times 5$ $+3 \times 2+3 \times 5) I=10+46 I$.

(4) $z_{1}^{2}=\left(x_{1}+y_{1} I\right)^{2}=x_{1}^{2}+\left(2 x_{1} y_{1}+y_{1}^{2}\right) I=5^{2}+(2 \times 5 \times 3$ $\left.+3^{2}\right) I=25+39 I, z_{2}^{2}=\left(x_{2}+y_{2} I\right)^{2}=x_{2}^{2}+\left(2 x_{2} y_{2}+y_{2}^{2}\right)$ $I=2^{2}+\left(2 \times 2 \times 5+5^{2}\right) \mathrm{I}=4+45 I$.

(5) $z_{1} / z_{2}=x_{1}+y_{1} I / x_{2}+y_{2} I=x_{1} / x_{2}+x_{2} y_{1}-\mathrm{x}_{1} \mathrm{y}_{2} / \mathrm{x}_{2}$ $\left(x_{2}+y_{2}\right) I=5 / 2+2 \times 3-5 \times 5 / 2(2+5) I=2.5-$ $1.3571 I$.

(6) $\sqrt{z_{1}}=\sqrt{x_{1}+y_{1} I}=\left\{\begin{array}{l}\sqrt{x_{1}}-\left(\sqrt{x_{1}}+\sqrt{x_{1}+y_{1}}\right) I \\ \sqrt{x_{1}}-\left(\sqrt{x_{1}}-\sqrt{x_{1}+y_{1}}\right) I \\ -\sqrt{x_{1}}+\left(\sqrt{x_{1}}+\sqrt{x_{1}+y_{1}}\right) I \\ -\sqrt{x_{1}}+\left(\sqrt{x_{1}}-\sqrt{x_{1}+y_{1}}\right) I\end{array}=\right.$

$\left\{\begin{array}{l}\sqrt{5}-(\sqrt{5}+\sqrt{5+3}) I=2.2361-5.0645 I \\ \sqrt{5}-(\sqrt{5}-\sqrt{5+3}) I=2.2361+0.5924 I \\ -\sqrt{5}+(\sqrt{5}+\sqrt{5+3}) I=-2.2361+5.0645 I \\ -\sqrt{5}+(\sqrt{5}-\sqrt{5+3}) I=-2.2361-0.5924 I\end{array}\right.$

$\sqrt{z_{2}}=\sqrt{x_{2}+y_{2} I}=\left\{\begin{array}{l}\sqrt{x_{2}}-\left(\sqrt{x_{2}}+\sqrt{x_{2}+y_{2}}\right) I \\ \sqrt{x_{2}}-\left(\sqrt{x_{2}}-\sqrt{x_{2}+y_{2}}\right) I \\ -\sqrt{x_{2}}+\left(\sqrt{x_{2}}+\sqrt{x_{2}+y_{2}}\right) I \\ -\sqrt{x_{2}}+\left(\sqrt{x_{2}}-\sqrt{x_{2}+y_{2}}\right) I\end{array}=\right.$

$\left\{\begin{array}{l}\sqrt{2}-(\sqrt{2}+\sqrt{2+5}) I=1.4142-4.0600 I \\ \sqrt{2}-(\sqrt{2}-\sqrt{2+5}) I=1.4142+1.2315 I \\ -\sqrt{2}+(\sqrt{2}+\sqrt{2+5}) I=-1.4142+4.0600 I \\ -\sqrt{2}+(\sqrt{2}-\sqrt{2+5}) I=-1.4142-1.2315 I\end{array}\right.$. 


\section{Neutrosophic Number Nonlinear Programming (NN-NP)}

3.1. NN-NP Mathematical Model. The usual mathematical model of NN-NP is represented in the following form $[36,37]$ :

$$
\begin{aligned}
& \operatorname{Min} F(x, I) \\
& \text { s.t. } g_{i}(x, I) \leq c_{i}, \quad i=1,2, \ldots, p, \\
& h_{j}(x, I)=0, \quad j=1,2, \ldots, q, \\
& x \in Z^{n},
\end{aligned}
$$

where $\quad g_{1}(x, I), g_{2}(x, I), \ldots, g_{p}(x, I), h_{1}(x, I), h_{2}(x, I)$ : $Z^{n} \longrightarrow \boldsymbol{Z}\left(\boldsymbol{Z}\right.$ is the set of all NNs), and $I \in\left[I^{L}, I^{U}\right]$ (the interval range of $I)$.

\subsection{Inventory Mathematical Model [38]}

3.2.1. Notations. The following notations are used in the inventory model.

Three decision variables:
(i) $D$ : demand/unit/time
(ii) $Q_{p}$ : production quantity/batch
(iii) $C_{s}$ : setup cost/unit/time

Except the above cost variable CS, three other cost variables are
(i) $C_{t a}$ : total average cost/unit/time
(ii) $C_{t p}$ : total production cost/cycle
(iii) $C_{h}$ : time depending on holding cost/unit/item

Other time and space variables:
(i) $T$ : every cycle of length
(ii) $Q(t)$ : inventory level at time $t(t \geq 0)$
(iii) $S$ : total storage space area
(iv) $s_{0}$ : space area/unit/quantity.

3.2.2. Assumptions. The inventory model is developed by considering the following assumptions:

(i) Only one item is involved in the inventory system.

(ii) The replenishment occurs with the near instantaneous response.

(iii) The startup time can be ignored.

(iv) The demand rate at any time is constant.

(v) The total production cost $C_{t p}$ is related to the setup cost CS and production quantity $Q_{P}$.

(vi) Holding cost is the time depended function.

3.3. Model Formation. As shown in Figure 1, in every time period $T$, the value of the production quantity $Q_{(t)}$ decreases from $Q_{p}$ to zero. The slope of the line is constant negative $D$ and denoted by $(d Q(t) / d t)=-D(0 \leq t \leq T)$.

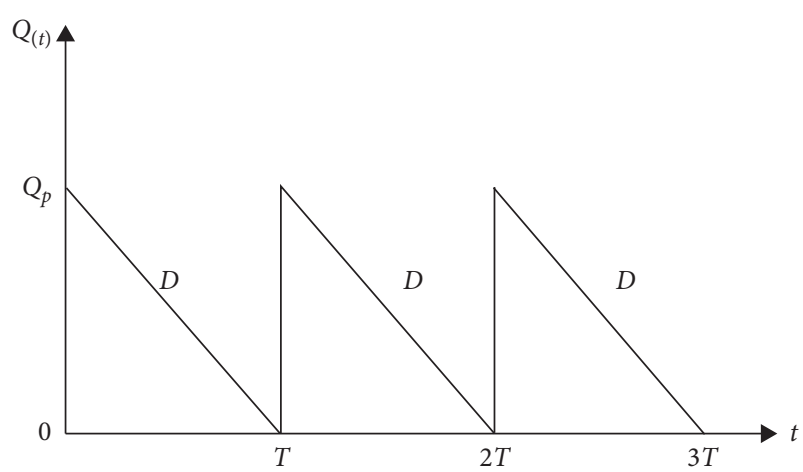

FIGURe 1: Crisp inventory model.

The total average cost of the cycle $T$ (denoted by $C_{t a}$ ) consists of three sections: setup cost (denoted by $C_{1}$ ), holding cost (denoted by $C_{2}$ ), and production cost (denoted by $C_{3}$ ).

$$
C_{t a}=C_{1}+C_{2}+C_{3} .
$$

Because we have the equation $Q(t)=Q_{p}-D t$, we obtain the cycle $T, T=\left(Q_{p} / D\right)$.

$$
\begin{aligned}
& C_{1}=\frac{C_{s}}{T}=\frac{C_{s} D}{Q_{p}}, \\
& C_{2}=\frac{\int_{0}^{T} C_{h} Q(t) d t}{T}=\frac{\int_{0}^{T} e t Q(t) d t}{T}=\frac{\left(e Q_{p}^{3} / 6 D^{2}\right)}{T} \\
& C_{3}=\frac{C_{t p}}{T}=\frac{f C_{s}^{-x} Q_{p}^{-y}}{T}=\frac{f D}{C_{s}^{x} Q_{p}^{1+y}} \frac{e Q_{p}^{2}}{6 D} .
\end{aligned}
$$

Based on equations (2) and (3), we obtain the following equation:

$$
C_{t a}=\frac{C_{s} D}{Q_{p}}+\frac{e Q_{p}^{2}}{6 D}+\frac{f D}{C_{s}^{x} Q_{p}^{1+y}}
$$

So, the inventory model is constructed as follows:

$$
\begin{aligned}
& \operatorname{Min} C_{t a}\left(D, C_{s}, Q_{p}\right)=\frac{C_{s} D}{Q_{p}}+\frac{e Q_{p}^{2}}{6 D}+\frac{f D}{C_{s}^{x} Q_{p}^{1+y}}, \\
& \text { s.t. } s_{0} Q_{p} \leq S, \\
& D, C_{s}, Q_{p}>0 .
\end{aligned}
$$

3.4. Solution Corresponding to Matlab Built-In Function "fmincon()". In order to conveniently calculate the solutions, we simplify some parameters and set some constants with history records, where $e=18, f=5, x=1, y=3$, $s_{0}=200$, and $S=1100$. When we assume $D=x_{1}, C_{s}=x_{2}$, and $Q_{p}=x_{3}$, we can obtain the following mathematical model: 
$\operatorname{Min} C_{t a}\left(x_{1}, x_{2}, x_{3}\right)=\frac{x_{1} x_{2}}{x_{3}}+\frac{18 x_{3}^{2}}{6 x_{1}}+\frac{5 x_{1}}{x_{2} x_{3}^{4}}$,

s.t. $200 x_{3} \leq 1100$ with maximum allowable tolerance

$$
400 x_{1}, x_{2}, x_{3} \geq 0 \text {. }
$$

Assume $a_{1}=x_{1}-40.496 I, a_{2}=x_{2}+0.058 I$, and $a_{3}=x_{3}-2 I$; then, equation (6) can be expressed in the following form:

s.t. $200(x 3-2 I) \geq 1100$ with maximum allowable tolerance 400 ,

$x_{1}-40.496 I>0$,

$x_{2}+0.058 I>0$,

$x_{3}-2 I>0$.

According to the de-neutrosophication technique proposed by Ye [39] and considering $I=0$ or 0.5 or 1 as the minimum or moderate or maximum indeterminacy, we can obtain three optimal solutions as follows:
(1) $x_{1}^{*}=80.615, \quad x_{2}^{*}=0.097, x_{3}^{*}=7.500$, and $f\left(x^{*}, I\right)$ $=4.187$ for $I=0$.

(2) $x_{1}^{*}=60.367, \quad x_{2}^{*}=0.126, \quad x_{3}^{*}=6.5$, and $f\left(x^{*}, I\right)$ $=4.343$ for $I=0.5$.

(3) $x_{1}^{*}=40.119, \quad x_{2}^{*}=0.155, \quad x_{3}^{*}=5.5, \quad$ and $f\left(x^{*}, I\right)$ $=4.525$ for $I=1$.

Clearly, using the indeterminacy $I \in[0,1]$, different optimal results are revealed. The optimal solutions of the optimization problem are $x_{1}^{*}=[40.119,80.615], x_{2}^{*}=[0.097$, $0.155]$, and $x_{3}^{*}=[5.5,7.5]$ for $f\left(x^{*}, I\right)=[4.187,4.525]$, which show the interval optimal ranges.

3.5. Solution Corresponding to Operations of NNs. According to the front optimal solutions, we assume $a_{1}=x_{1}+y_{1} I=80.615-40.496 \mathrm{I}, a_{2}=x_{2}+y_{2} I=0.097+0.058 I$, and $a_{3}=x_{3}-y_{3} I=7.500-2 I$, and then we give the results by equation (9):

$$
\begin{aligned}
& \operatorname{Min} C_{t a}\left(a_{1}, a_{2}, a_{3}\right)=\frac{a_{1} a_{2}}{a_{3}}+\frac{18 a_{3}^{2}}{6 a_{1}}+\frac{5 a_{1}}{a_{2} a_{3}^{4}}=\frac{\left(x_{1}+y_{1} I\right)\left(x_{2}+y_{2} I\right)}{x_{3}+y_{3} I}+\frac{18\left(x_{3}+y_{3} I\right)^{2}}{6\left(x_{1}+y_{1} I\right)}+\frac{5\left(x_{1}+y_{1} I\right)}{\left(x_{2}+y_{2} I\right)\left(x_{3}+y_{3} I\right)^{4}} \\
& =\frac{x_{1} x_{2}+\left(x_{1} y_{2}+x_{2} y_{1}+y_{1} y_{2}\right) I}{x_{3}+y_{3} I}+\frac{3\left[x_{3}^{2}+\left(2 x_{3} y_{3}+y_{3}^{2}\right) I\right]}{\left(x_{1}+y_{1} I\right)}+\frac{5\left(x_{1}+y_{1} I\right)}{\left(x_{2}+y_{2} I\right)\left[x_{3}^{2}+\left(2 x_{3} y_{3}+y_{3}^{2}\right) I\right]^{2}} \\
& =\frac{x_{1} x_{2}+\left(x_{1} y_{2}+x_{2} y_{1}+y_{1} y_{2}\right) I}{x_{3}+y_{3} I}+\frac{3\left[x_{3}^{2}+\left(2 x_{3} y_{3}+y_{3}^{2}\right) I\right]}{\left(x_{1}+y_{1} I\right)} \\
& +\frac{5\left(x_{1}+y_{1} I\right)}{\left(x_{2}+y_{2} I\right)\left\{x_{3}^{4}+\left[2\left(2 x_{3} y_{3}+y_{3}^{2}\right) x_{3}^{2}+\left(2 x_{3} y_{3}+y_{3}^{2}\right)^{2}\right] I\right\}}
\end{aligned}
$$

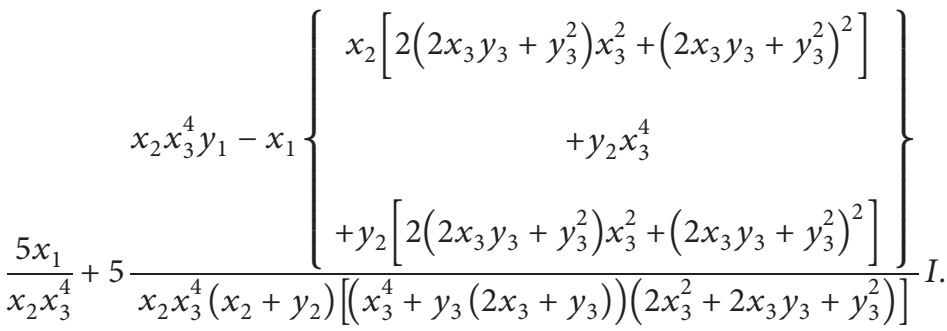

Because $a_{1}=80.615-40.496 I, a_{2}=0.097+0.058 I$, and $a_{3}=7.500-2 I$, we can get $x_{1}=80.615, y_{1}=-40.496$, $x_{2}=0.097, y_{2}=0.058, x_{3}=7.5$, and $y_{3}=-2$. Then, we calculate the three costs, respectively, as follows:

Setup cost:

$$
C_{1}=\frac{\left(x_{1}+y_{1} I\right)\left(x_{2}+y_{2} I\right)}{x_{3}+y_{3} I}=1.407+0.087 I .
$$

Holding cost:

$$
C_{2}=\frac{18\left(x_{3}+y_{3} I\right)^{2}}{6\left(x_{1}+y_{1} I\right)}=2.093+0.169 I
$$

Production cost:

$$
C_{3}=\frac{5\left(x_{1}+y_{1} I\right)}{\left(x_{2}+y_{2} I\right)\left(x_{3}+y_{3} I\right)^{4}}=1.047+0.082 I
$$

Then, we add the three costs and obtain the total cost $C_{t a}$ with equation (2) as follows: 


$$
\begin{aligned}
C_{t a}= & C_{1}+C_{2}+C_{3}=\frac{\left(x_{1}+y_{1} I\right)\left(x_{2}+y_{2} I\right)}{x_{3}+y_{3} I}+\frac{18\left(x_{3}+y_{3} I\right)^{2}}{6\left(x_{1}+y_{1} I\right)} \\
& +\frac{5\left(x_{1}+y_{1} I\right)}{\left(x_{2}+y_{2} I\right)\left(x_{3}+y_{3} I\right)^{4}} \\
= & (1.047+0.087 I)+(2.093+0.169 I)+(1.047+0.082 I) \\
= & 4.187+0.338 I .
\end{aligned}
$$

So, the calculational results validate that the same solution is obtained by using the two methods of both the Matlab built-in function "fmincon" and the operations of NNs, which are $x_{1}^{*}=[40.119,80.615], x_{2}^{*}=[0.097,0.155]$, and $x_{3}^{*}=[5.5,7.5]$ for $f\left(x^{*}, I\right)=[4.187,4.525]$. We also obtain every cost $C_{1}=[1.047,1.134], C_{2}=[2.093,2.262]$, and $C_{3}=[1.047,1.129]$, which are the interval optimal ranges.

\section{Production Planning Mathematical Model}

4.1. NN-LP Mathematical Model. The usual mathematical model of NN-LP is similar to mathematical model (1), so we omit it.

\subsection{Production Planning Mathematical Model}

4.2.1. Notations. The following notations are used in the production planning model.

Nine decision variables:

(i) $a_{1}$ to $a_{6}$ : product quantities of six plans of type I

(ii) $a_{7}$ to $a_{8}$ : product quantities of two plans of type II

(iii) $a_{9}$ : product quantities of two plans of type III

Objective function:

(i) $b$ : maximum profit

4.2.2. Assumptions. The production planning model is developed by considering the following assumptions:

(i) Every product must pass two working procedures: A and $B$. (ii) The startup time of two working procedures can be ignored.

(iii) Product quantities are only affected by validity time of machines.

(iv) The demand rate at any time is constant.

4.3. Model Formation. As shown in Table 1, we consider an application in production planning studied by $\mathrm{Hu}$ [40]. A company manufactures three types of products: Types I, II, and III. All types must pass two working procedures: $A$ and $B$. We consider that procedure $A$ can be operated on machine $A_{1}$ or $A_{2}$ and procedure $B$ can be operated on the machines $B_{1}, B_{2}$, and $B_{3}$. Type I can be operated on all machines of procedure $A$ and procedure $B$; Type II can be operated on all machines of procedure $A$ and only machine $B_{1}$ of procedure $B$; Type III can be operated on only machine $A_{2}$ of procedure $A$ and machine $B_{2}$ of procedure $B$. Our aim is to schedule the optimal production planning, which can pursue for the maximum profits. All used data are listed in Table 1, including required procedure time of every working procedure, processing fees, material cost, and selling price per unit. So, Type I has six plans to produce products, along with $\left(A_{1}, B_{1}\right)$ or $\left(A_{1}, B_{2}\right)$ or $\left(A_{1}, B_{3}\right)$ or $\left(A_{2}, B_{1}\right)$ or $\left(A_{2}, B_{2}\right)$ or $\left(A_{2}, B_{3}\right)$, respectively. Similarly, we consider the product quantities of the six plans $a_{1}, a_{2}, a_{3}, a_{4}, a_{5}$, and $a_{6}$, respectively. Type II has two plans to produce products, along with $\left(A_{1}, B_{1}\right)$ or $\left(A_{2}, B_{1}\right)$, and Type III has one plan to produce products, along with $\left(A_{2}, B_{2}\right)$. We consider the product quantities of the remaining three plans $a_{7}, a_{8}$, and $a_{9}$, respectively. So, we can get the following objective function.

$$
\begin{aligned}
b= & {[(1.20+0.03 I)-(0.23+0.03 I)] \times\left(a_{1}+a_{2}+a_{3}+a_{4}+a_{5}+a_{6}\right)+[(1.60+0.5 I)-(0.30+0.07 I)] } \\
& \times\left(a_{7}+a_{8}\right)+[(2.30+0.3 I)-(0.30+0.05 I)] \times a 9-(0.04+0.02 I) \times\left[(4.5+1.7 I) \times\left(a_{1}+a_{2}+a_{3}\right)+(8+I) \times a_{7}\right] \\
& -(0.02+0.01 I) \times\left[(0.67+0.8 I) \times\left(a_{4}+a_{5}+a_{6}\right)+(8.6+1.4 I) \times a_{8}+(11-I) \times a_{9}\right]-(0.05+0.02 I) \\
& \times\left[(5.6-0.1 I) \times(a 1+a 4)+(7.8+1.2 I) \times\left(a_{7}+a_{8}\right)\right]-(0.10+0.02 I) \times\left[(3.5+2.5 I) \times\left(a_{2}+a_{5}\right)+(10+2 I) \times a_{9}\right] \\
& -(0.04+0.02 I) \times\left[(6.7+1.3 I) \times\left(a_{3}+a_{6}\right)\right] .
\end{aligned}
$$

So, we get the followed production planning mathematical model: 
TABLE 1: All data of three types of products.

\begin{tabular}{|c|c|c|c|c|c|}
\hline \multirow{2}{*}{ Machine } & \multicolumn{3}{|c|}{ Product } & \multirow{2}{*}{ Validity time (hours/machine) } & \multirow{2}{*}{ Processing fees ( $\$ /$ hour/machine) } \\
\hline & I & II & III & & \\
\hline$A_{1}$ & $4.5+1.7 I$ & $8+I$ & - & $5600+700 I$ & $0.04+0.02 I$ \\
\hline$A_{2}$ & $6.7+1.8 I$ & $8.6+1.4 I$ & $11-I$ & $9000+700 I$ & $0.02+0.01 I$ \\
\hline$B_{1}$ & $5.6-0.1 I$ & $7.8+1.2 I$ & - & $3700+1100 I$ & $0.05+0.02 I$ \\
\hline $\mathrm{B}_{2}$ & $3.5+2.5 I$ & - & $10+2 I$ & $6000+1000 I$ & $0.10+0.02 I$ \\
\hline$B_{3}$ & $6.7+1.3 I$ & - & - & $3500+1500 I$ & $0.04+0.02 I$ \\
\hline Material cost (\$/piece) & $0.23+0.03 I$ & $0.30+0.07 I$ & $0.30+0.05 I$ & \multirow{2}{*}{-} & \multirow[b]{2}{*}{ - } \\
\hline Selling price (\$/piece) & $1.20+0.03 I$ & $1.60+0.5 I$ & $2.30+0.3 I$ & & \\
\hline
\end{tabular}

$$
\begin{array}{ll}
\max & b \\
\text { s.t. } \quad & (4.5+1.71) \times\left(x_{1}+x_{2}+x_{3}\right)+(8+I) \times x 7 \leq 5600+700 I \\
& (6.7+1.8 I) \times\left(x_{4}+x_{5}+x_{6}\right)+(8.6+1.4 I) \times x_{8}+(11-I) \times x_{9} \leq 0.02+0.01 I, \\
& (5.6-0.1 I) \times\left(x_{1}+x_{4}\right)+(7.8+1.2 I) \times\left(x_{7}+x_{8}\right) \leq 0.05+0.02 I \\
& (3.5+2.5 I) \times\left(x_{2}+x_{5}\right)+(10+2 I) \times x_{9} \leq 0.10+0.02 I \\
& (6.7+1.3 I) \times\left(x_{3}+x_{6}\right) \leq 0.04+0.02 I
\end{array}
$$

4.4. Solution regarding Matlab Built-In Function "fmincon()". According to the de-neutrosophication technique proposed by $\mathrm{Ye}$ [37] and considering $I=0$ or 0.5 or 1 as the minimum or moderate or maximum indeterminacy, we can obtain three optimal solutions as follows:

(1) $x_{1}^{*}=0, \quad x_{2}^{*}=778.508, \quad x_{3}^{*}=465.936, \quad x_{4}^{*}=0, \quad x_{5}^{*}=$ $677.953, x_{6}^{*}=56.452, x_{7}^{*}=0, x_{8}^{*}=474.359, x_{9}^{*}=0$, and $f\left(x^{*}, I\right)=1297.389$ for $I=0$.

(2) $x_{1}^{*}=0, x_{2}^{*}=0, x_{3}^{*}=578.231, x_{4}^{*}=0, x_{5}^{*}=0, x_{6}^{*}=0$, $x_{7}^{*}=167.732, \quad x_{8}^{*}=338.221, \quad x_{9}^{*}=590.909, \quad$ and $f\left(x^{*}, I\right)=940.871$ for $I=0.5$.

(3) $x_{1}^{*}=0, x_{2}^{*}=0, x_{3}^{*}=625, x_{4}^{*}=0, x_{5}^{*}=0, x_{6}^{*}=0, x_{7}^{*}=$ $146.667, x_{8}^{*}=386.667, x_{9}^{*}=583.333$, and $f\left(x^{*}, I\right)$ $=762.717$ for $I=1$.

Clearly, using the indeterminacy $I \in[0,1]$, different optimal results are revealed. The optimal solutions of the optimization problem are $x_{1}^{*}=[0,0], x_{2}^{*}=[0,778.508]$, $x_{3}^{*}=[465.936,625], x_{4}^{*}=[0,0], x_{5}^{*}=[0,677.953], x_{6}^{*}=[0$, $56.452], x_{7}^{*}=[0,146.667], x_{8}^{*}=[386.667,474.359]$, and $x_{9}^{*}=$ $[0,583.333]$ for $f\left(x^{*}, I\right)=[762.717,1297.389]$, which shows the interval optimal ranges.

4.5. Solution regarding Operations of NNs. According the front optimal solutions, we next calculate the nine relation formulas of the indeterminacy I and variables $a_{1}, a_{2}, a_{3}, a_{4}$, $a_{5}, a_{6}, a_{7}, a_{8}$, and $a_{9}$. For example, let us calculate $a_{3}=465.936+159.064 I$. Firstly, according to three points $(0$, 465.936), $(0.5,578.231)$, and $(1,625)$, we obtain the linear equation $\left(a_{3}=159.06 I+476.86\right)$. Next we amend the intercept of trend curve on the vertical coordinate. The other linear equations are obtained in the same way. So, $a_{1}=x_{1}+y_{1} I=0+0 I=0, \quad a_{2}=x_{2}+y_{2} I=778.508-778.508 I$, $a_{3}=x_{3}+y_{3} I=465.936+159.064 I, \quad a_{4}=x_{4}+y_{4} I=0+0 I=0$, $a_{5}=x_{5}+y_{5} I=677.953-677.953 I, \quad a_{6}=x_{6}+y_{6} \mathrm{I}=56.452$ $-56.452 I, \quad a_{7}=x_{7}-y_{7} I=0+146.667 I, \quad a_{8}=x_{8}+y_{8} I=474.359$ $-87.692 I$, and $a_{9}=x_{9}+y_{9} I=0+583.333 I$; then, we calculate the results of equation (13) as follows:

$b=[(1.2+0.03 I)-(0.23+0.03 I)] \times\left(a_{1}+a_{2}+a_{3}+a_{4}+a_{5}\right.$ $\left.+a_{6}\right)+[(1.60+0.5 I)-(0.30+0.07 I)] \times\left(a_{7}+a_{8}\right)+[(2.30+$ $0.3 I)-(0.30+0.05 I)] \times a_{9}-(0.04+0.02 I) \times[(4.5+1.7 I) \times$ $\left.\left(a_{1}+a_{2}+a_{3}\right)+(8+I) \times a_{7}\right]-(0.02+0.01 I) \times[(6.7+1.8 I) \times$ $\left.\left(a_{4}+a_{5}+a_{6}\right)+(8.6+1.4 I) \times a_{8}+(11-I) \times a_{9}\right]-(0.05+$ $0.02 I) \times\left[(5.6-0.1 I) \times\left(a_{1}+a_{4}\right)+(7.8+1.2 I) \times\left(a_{7}+a_{8}\right)\right]-$ $(0.10+0.02 I) \times\left[(3.5+2.5 I) \times\left(a_{2}+a_{5}\right)+(10+2 I) \times a_{9}\right]-$ $(0.04+0.02 I) \times\left[(6.7+1.3 I) \times\left(a_{3}+a_{6}\right)\right]=0.97 \times\left(a_{1}+a_{2}+\right.$ $\left.a_{3}+a_{4}+a_{5}+a_{6}\right)+(1.30+0.43 I) \times\left(a_{7}+a_{8}\right)+(2.0+0.25 I) \times$ $a_{9}-(0.04+0.02 I) \times\left[(4.5+1.7 I) \times\left(a_{1}+a_{2}+a_{3}\right)+(8+\mathrm{I}) \times\right.$ $\left.a_{7}\right]-(0.02+0.01 I) \times\left[(6.7+1.8 I) \times\left(a_{4}+a_{5}+a_{6}\right)+(8.6+\right.$ $\left.1.4 I) \times a_{8}+(11-I) \times a_{9}\right]-(0.05+0.02 I) \times\left[(5.6-0.1 \mathrm{I}) \times\left(a_{1}\right.\right.$ $\left.\left.+a_{4}\right)+(7.8+1.2 I) \times\left(a_{7}+a_{8}\right)\right]-(0.10+0.02 I) \times[(3.5+2.5 I)$ $\left.\times\left(a_{2}+a_{5}\right)+(10+2 I) \times a_{9}\right]-(0.04+0.02 I) \times[(6.7+1.3 I) \times$ $\left.\left(a_{3}+a_{6}\right)\right]=0.97 \times\left(a_{2}+a_{3}+a_{5}+a_{6}\right)+(1.30+0.43 I) \times\left(a_{7}+\right.$ $\left.a_{8}\right)+(2.0+0.25 I) \times a_{9}-(0.04+0.02 I) \times\left[(4.5+1.7 I) \times\left(a_{2}+\right.\right.$ $\left.\left.a_{3}\right)+(8+I) \times a_{7}\right]-(0.02+0.01 I) \times\left[(6.7+1.8 I) \times\left(a_{5}+a_{6}\right)+\right.$ $\left.(8.6+1.4 I) \times a_{8}+(11-I) \times a_{9}\right]-(0.05+0.02 I) \times[(7.8+1.2 I)$ $\left.\times\left(a_{7}+a_{8}\right)\right]-(0.10+0.02 \mathrm{I}) \times\left[(3.5+2.5 I) \times\left(a_{2}+a_{5}\right)+(10+\right.$ $\left.2 I) \times a_{9}\right]-(0.04+0.02 I) \times\left[(6.7+1.3 I) \times\left(a_{3}+a_{6}\right)\right]=0.97 \times$ $(778.508-778.508 I+465.936+159.064 I+677.953-$ $677.953 I+56.452-56.452 I)+(1.30+0.43 I) \times(0+146.667 I$ $+474.359-87.692 I)+(2.0+0.25 I) \times(0+583.333 I)-(0.04$ $+0.02 I) \times[(4.5+1.7 I) \times(778.508-778.508 I+465.936+$ $159.064 I)+(8+\mathrm{I}) \times(0+146.667 I)]-(0.02+0.01 I) \times[(6.7+$ $1.8 I) \times(0+677.953-677.953 I+56.452-56.452 I)+(8.6+$ $1.4 I) \times(474.359-87.692 I)+(11-I) \times(0+583.333 I)]-$ $(0.05+0.02 \mathrm{I}) \times[(7.8+1.2 I) \times(0+146.667 I+474.359-$ $87.692 I)]-(0.10+0.02 \mathrm{I}) \times[(3.5+2.5 \mathrm{I}) \times(778.508-$ $778.508 I+677.953-677.953 I)+(10+2 I) \times(0+583.333 I)]$ $-(0.04+0.02 I) \times[(6.7+1.3 I) \times(465.936+159.064 I+$ 
$56.452-56.452 I)]=0.97 \times(1978.849-1353.849 I)+(1.3+$ $0.43 I) \times(474.359+58.975 I)+(2+0.25 I) \times(0+583.333 I)-$ $(0.04+0.02 I) \times(5599.998-404.995 I)-(0.02+0.01 I) \times$ $(9000.001+699.9991 I)-(0.05+0.02 I) \times(3700+1100.006 I)$ $-(0.10+0.02 \mathrm{I}) \times(5097.614+1902.383 I)-(0.04+0.02 \mathrm{I}) \times$ $(3500+1500 I)=1919.484-1313.234 I+616.6667+306.001 I$ $+1312.499 I-224-87.7 I-180-111.000 I-185-509.761$ $-330.238 I-140-160.000 I=1297.389-534.672 I$.

So, these calculational results validate that the same solution is obtained by using the two methods of both the Matlab built-in function "fmincon()" and the operations of NNs, which are $x_{1}^{*}=[0,0], x_{2}^{*}=[0,778.508], x_{3}^{*}=[465.936$, $625], x_{4}^{*}=[0,0], x_{5}^{*}=[0,677.953], x_{6}^{*}=[0,56.452], x_{7}^{*}=[0$, $146.667], x_{8}^{*}=[386.667,474.359]$, and $x_{9}^{*}=[0,583.333]$ for $f\left(x^{*}, I\right)=[762.717,1297.389]$ and show the interval optimal ranges.

\section{Conclusion}

This paper first introduced some concepts and their operations of NNs with indeterminacy $I$. Next, we built a mathematical model with constrained conditions and then constructed the corresponding inventory model and production planning model. Finally, we obtained the optimal solutions by using the two methods of the Matlab built-in function "fmincon()" and the operations of NNs to solve the NN-NP and NN-LP problems with constrained conditions as preliminary application study in indeterminate setting. The final results show that the two methods obtained the same effective solutions, but the former needs the Matlab built-in function along with the simple calculational process, while the latter needs a lot of operations of NNs along with the complex calculational process. Some contributions in this study are that (1) different methods can obtain the same optimal results, (2) the NN-NP and NN-LP methods provided the new application ways for engineering management, (3) the NN-NP and NN-LP methods are more suitable than other ones under uncertain environments as the generalization of traditional programming methods, and (4) the two approaches can obtain the interval solutions for avoiding determinate solutions of traditional programming methods.

Obviously, the proposed NN-LP and NN-NP methods can handle indeterminate and/or determinate mathematical programming problems, which are the generalization of existing uncertain or certain linear and nonlinear programming methods. As the preliminary application study in this paper, however, there exist a lot of mathematical solution methods and proof problems along with some complexity/difficulty in the nonlinear programming problems which need to be studied further. Hence, as our future works, one is to further analyze the two presented methods of this paper from the mathematical problems, such as the convexity problem in the nonlinear programming, the stability and solution range problems regarding the changeability of NNs, and the sensitivities of NNs on the solution results, and then NN-LP and NN-NP approaches will be extended to other fields, such as engineering design and management science.

\section{Data Availability}

The data used to support the findings of this study are included within the article.

\section{Conflicts of Interest}

The authors declare that they have no conflicts of interest.

\section{References}

[1] S. Kumar, N. Kumar, and S. Liu, "An inventory model for deteriorating items under inflation and permissible delay in payments by genetic algorithm," Cogent Business \& Management, vol. 3, no. 1, 2016.

[2] A. Oliveira, T. Oliveira, and A. Seijasmacías, "The uniform distribution product: an approach to the $(\mathrm{Q}, \mathrm{r})$ inventory model using R," Journal of Applied Statistics, vol. 45, no. 2, pp. 284-297, 2018

[3] P. Mahata, G. C. Mahata, and S. Kumar De, "Optimal replenishment and credit policy in supply chain inventory model under two levels of trade credit with time- and creditsensitive demand involving default risk," Journal of Industrial Engineering International, vol. 14, no. 1, pp. 31-42, 2018.

[4] I. D. Wangsa and M. W. Hui, "An integrated vendor-buyer inventory model with transportation cost and stochastic demand," International Journal of Systems Science, vol. 5, no. 4, pp. 295-309, 2018.

[5] C. P. T. Hedenstierna and S. M. Disney, "Avoiding the capacity cost trap: three means of smoothing under cyclical production planning," International Journal of Production Economics, vol. 201, pp. 149-162, 2018.

[6] H.-M. Cho and I.-J. Jeong, "A two-level method of production planning and scheduling for bi-objective reentrant hybrid flow shops," Computers \& Industrial Engineering, vol. 106, pp. 174-181, 2017.

[7] C. Jiang, Z. G. Zhang, Q. F. Zhang, X. Han, H. C. Xie, and J. Liu, "A new nonlinear interval programming method for uncertain problems with dependent interval variables," $E$ uropean Journal of Operational Research, vol. 238, no. 1, pp. 245-253, 2014.

[8] I. Pergher and A. T. De Almeida, "A multi-attribute decision model for setting production planning parameters," Journal of Manufacturing Systems, vol. 42, pp. 224-232, 2017.

[9] G. S. Veresnikov, L. A. Pankova, and V. A. Pronina, "Uncertain programming in preliminary design of technical systems with uncertain parameters," Procedia Computer Science, vol. 103, pp. 36-43, 2017.

[10] C. Jiang, X. Y. Long, X. Han, Y. R. Tao, and J. Liu, "Probability-interval hybrid reliability analysis for cracked structures existing epistemic uncertainty," Engineering Fracture Mechanics, vol. 112-113, pp. 148-164, 2013.

[11] B. Zhang and J. Peng, "Uncertain programming model for uncertain optimal assignment problem," Applied Mathematical Modelling, vol. 37, no. 9, pp. 6458-6468, 2013.

[12] B. D. Liu and X. W. Chen, "Uncertain multiobjective programming and uncertain goal programming," Journal of Uncertainty Analysis and Applications, vol. 3, p. 10, 2015.

[13] L. Chen, J. Peng, and B. Zhang, "Uncertain goal programming models for bicriteria solid transportation problem," Applied Soft Computing, vol. 51, pp. 49-59, 2017.

[14] L. Wang, Z. Chen, G. Yang, Q. Sun, and J. Ge, "An interval uncertain optimization method using back-propagation 
neural network differentiation," Computer Methods in Applied Mechanics and Engineering, vol. 366, p. 113065, 2020.

[15] L. Wang, Z. Chen, and G. Yang, "An interval uncertainty analysis method for structural response bounds using feedforward neural network differentiation," Applied Mathematical Modelling, vol. 82, pp. 449-468, 2020.

[16] F. Smarandache, Neutrosophy: Neutrosophic Probability, Set, and Logic, American Research Press, Rehoboth, MA, USA, 1998.

[17] F. Smarandache, Introduction to Neutrosophic Statistics, Sitech \& Education Publishing, Columbus, OH, USA, 2014.

[18] F. Smarandache, Introduction to Neutrosophic Measure, Neutrosophic Integral, and Neutrosophic Probability, Sitech \& Education Publisher, Columbus, OH, USA, 2013.

[19] L. Kong, Y. Wu, and J. Ye, "Misfire fault diagnosis method of gasoline engines using the cosine similarity measure of neutrosophic numbers," Neutrosophic Sets and Systems, vol. 8, pp. 43-46, 2015.

[20] J. Ye, "Fault diagnoses of steam turbine using the exponential similarity measure of neutrosophic numbers," Journal of Intelligent \& Fuzzy Systems, vol. 30, no. 4, pp. 1927-1934, 2016.

[21] J. Ye, "Multiple-attribute group decision-making method under a neutrosophic number environment," Journal of Intelligent Systems, vol. 25, no. 3, pp. 377-386, 2016.

[22] J. Ye, "Bidirectional projection method for multiple attribute group decision making with neutrosophic numbers," Neural Computing and Applications, vol. 28, no. 5, pp. 1021-1029, 2017.

[23] F. Smarandache, Neutrosophic Precalculus and Neutrosophic Calculus, EuropaNova, Brussels, Belgium, 2015.

[24] J. Ye, R. Yong, Q. F. Liang, M. Huang, and S. G. Du, "Neutrosophic functions of the joint roughness coefficient (JRC) and the shear strength: a case study from the pyroclastic rock mass in Shaoxing City, China," Mathematical Problems in Engineering, vol. 2016, Article ID 4825709, , 2016.

[25] J. Ye, J. Chen, R. Yong, and S. Du, "Expression and analysis of joint roughness coefficient using neutrosophic number functions," Information, vol. 8, no. 2, p. 69, 2017.

[26] J. Chen, J. Ye, S. Du, and R. Yong, "Scale effect and anisotropy analyzed for neutrosophic numbers of rock joint roughness coefficient based on neutrosophic statistics," Symmetry, vol. 9, no. 10, p. 208, 2017.

[27] J. Chen, J. Ye, S. Du, and R. Yong, "Expressions of rock joint roughness coefficient using neutrosophic interval statistical numbers," Symmetry, vol. 9, no. 7, p. 123, 2017.

[28] J. Ye, "Neutrosophic linear equations and application in traffic flow problems," Algorithms, vol. 10, no. 4, p. 133, 2017.

[29] J. Ye, "Aggregation operators of neutrosophic linguistic numbers for multiple attribute group decision making," vol. 5, no. 1, , p. 1691, Springer Plus, 2016.

[30] Z. Fang and J. Ye, "Multiple attribute group decision-making method based on linguistic neutrosophic numbers," Symmetry, vol. 9, no. 7, p. 111, 2017.

[31] J. Ye, "Multiple attribute decision-making methods based on the expected value and the similarity measure of hesitant neutrosophic linguistic numbers," Cognitive Computation, vol. 10, no. 2, pp. 454-463, 2018.

[32] J. Ye, "Neutrosophic number linear programming method and its application under neutrosophic number environments," Soft Computing, vol. 22, no. 14, pp. 4639-4646, 2018.

[33] W. Jiang and J. Ye, "Optimal design of truss structures using a neutrosophic number optimization model under an indeterminate environment," Neutrosophic Sets and Systems, vol. 14, pp. 93-97, 2016.

[34] F. Smarandache, Introduction to Neutrosophic Measure, Neutrosophic Integral, and Neutrosophic Probability, Sitech \& Education Publishing, Craiova, Romania, 2013.

[35] F. Smrandache, Introduction to Neutrosophic Statistics, Sitech \& Education Publishing, Craiova, Romania, 2014.

[36] J. Ye, "Neutrosophic number linear programming method and its application under neutrosophic number environments," Soft Computing, vol. 22, no. 14, pp. 4639-4646, 2018.

[37] J. Ye, W. Cui, and Z. Lu, "Neutrosophic number nonlinear programming problems and their general solution methods under neutrosophic number environments," Axioms, vol. 7, p. 13, 2018.

[38] C. Kar, B. Mondal, and T. K. Roy, "An inventory model under space constraint in neutrosophic environment: a neutrosophic geometric programming approach," Neutrosophic Sets and Systems, vol. 21, pp. 93-109, 2018.

[39] J. Ye, "An improved neutrosophic number optimization method for optimal design of truss structures," New Mathematics and Natural Computation, vol. 14, no. 3, pp. 295-305, 2018.

[40] Y. Hu and Y. Guo, Operations Research Tutorial, Tsinghua University Press, Beijing, China, Fourth edition, 2012. 